Research article

\title{
Hemispheric dorsolateral prefrontal cortex lateralization in the regulation of empathy for pain
}

\author{
Gabriel G. Rêgo ${ }^{\mathrm{a}}$, Olívia M. Lapenta ${ }^{\mathrm{a}}$, Lucas M. Marques ${ }^{\mathrm{a}}$, Thiago L. Costa ${ }^{\mathrm{a}}$, Jorge Leite ${ }^{\mathrm{b}, \mathrm{d}}$, \\ Sandra Carvalho ${ }^{\mathrm{b}, \mathrm{d}}$, Óscar F. Gonçalves ${ }^{\mathrm{b}, \mathrm{d}}$, André R. Brunoni ${ }^{\mathrm{c}}$, Felipe Fregni ${ }^{\mathrm{e}}$, \\ Paulo S. Boggio ${ }^{\text {a,* }}$ \\ a Social and Cognitive Neuroscience Laboratory and Developmental Disorders Program, Center for Health and Biological Sciences, Mackenzie Presbyterian \\ University, 01241-001 Sao Paulo, Brazil \\ ${ }^{\mathrm{b}}$ Bouve College of Health Sciences, Department of Counseling and Applied Educational Psychology, Northeastern University, Boston, MA, USA \\ c Service of Interdisciplinary Neuromodulation (SIN), Department and Institute of Psychiatry and University Hospital, University of São Paulo, São Paulo, \\ Brazil \\ d Neuropsychophysiology Lab - CIPsi School of Psychology, University of Minho, Braga, Portugal \\ e Spaulding Neuromodulation Center, Spaulding Rehabilitation Hospital and Massachusetts General Hospital, Harvard Medical School, Boston, MA, USA
}

\section{H I G H L I G H T S}

- Neuromodulation of DLPFC excitability changes empathic affective responses.

- It is proposed a common role for left and right PFC in personal distress modulation.

- It is proposed distinct roles for lateralized DLPFC activity in cognitive empathy.

\section{A R T I C L E I N F O}

\section{Article history:}

Received 22 January 2015

Received in revised form 2 March 2015

Accepted 20 March 2015

Available online 21 March 2015

\section{Keywords:}

Physical pain

Pain empathy

Emotion regulation

Dorsolateral prefrontal cortex

Transcranial direct current stimulation

Pupil dilation

\begin{abstract}
A B S T R A C T
The dorsolateral prefrontal cortex (DLPFC) is involved in the cognitive appraisal and modulation of the pain experience. In this sham-controlled study, with healthy volunteers, we used bi-hemispheric transcranial direct current stimulation (tDCS) over the DLPFC to assess emotional reactions elicited by pain observation. Left-cathodal/right-anodal tDCS decreased valence and arousal evaluations compared to other tDCS conditions. Compared to sham condition, both left-cathodal/right-anodal and left-anodal/right-cathodal tDCS decreased hostility, sadness and self-pain perception. These decreased sensations after both active tDCS suggest a common role for left and right DLPFC in personal distress modulation. However, the differences in arousal and valence evaluations point to distinct roles of lateralized DLPFC in cognitive empathy, probably through distinct emotion regulation mechanisms.
\end{abstract}

(C) 2015 Elsevier Ireland Ltd. All rights reserved.

\section{Introduction}

Seeing other people experience pain, usually results in discomfort and distress. This affective response for pain has been investigated with neuroimaging studies [1]. These studies have shown the activation in brain areas such as the dorsolateral prefrontal cortex (DLPFC), the anterior cingulate cortex and the anterior insula $[1,2]$.

\footnotetext{
* Corresponding author. Tel.: +55 112114 8001; fax: +55 1121148001.

E-mail address: boggio@mackenzie.br (P.S. Boggio).
}

Both left and right DLPFC activity have been related to decreased self-pain perception [3,4], and this modulating seems, appears to be associated with its main function on general emotional regulation processes, such as cognitive reappraisal and attention modulation of affective responses [5]. According to Ochsner, Silver and Buhle (2012) [5], different emotional regulation strategies are associated with a lateralized DLPFC activity: while the left side is often involved with the meaning reinterpretation of the affective response, the right side seems to play a role on psychological distancing from the emotional stimulus.

One useful tool to increase our understanding of the role of DLPFC in one's affective reaction when seeing other's pain is transcranial direct current stimulation (tDCS). This simple, but effective 
method of neural modulation has been extensively used to assess cognitive processing [6,7]. The tDCS is characterized by the application of a low-intensity direct current through electrodes positioned on the scalp, where cortical activity under anodal electrode usually increases, whereas, it decreases under cathodal electrode [8].

Previous works with tDCS showed that, anodal stimulation over left DLPFC decreased self-reported responses of distress in volunteers seeing images of other people in pain [9]. However, the specific role of lateralized DLPFC in modulating the affective response to observing someone experiencing pain is still not clear. Given this, we aimed here to investigate the lateralized DLPFC role in affective pain empathy using a bifrontal TDCS montage, that would allow modifying left and right DLPFC areas in the opposite manner, during an emotional-laden movies visualization portraying people under painful situations. The motivation for this study was to understand further the specific contribution of DLPFC in the processing of several behavioral domains during affective pain empathy process. It has been shown that the affective areas of the pain matrix are the major neural areas involved when observing the experience of others in pain [2]. Nevertheless, the majority of studies investigating the lateralized aspects of DLPFC on emotion regulation and pain processing use of correlational techniques, leaving unexplained the causal role of such structures in these processes.

Given, the role of both left and right DLPFC on emotion regulation [5] and in decreased self-pain perception [3,4], we hypothesized that tDCS would diminish the impact of movies portraying people in pain, as expressed by self-reports of emotional valence and arousal of the videos, as well as self-reports of changes in mood and self-pain perception.

Finally, to provide physiological complementary information to the behavioral data, we include collected pupil dilation responses. Through its variations, it might be possible to clarify distinct cognitive engagement due to different lateralized DLPFC stimulation [10]. We expected shorter dilation after tDCS in comparison to sham condition.

\section{Material and methods}

\subsection{Participants}

Twenty-four healthy students of Mackenzie Presbyterian University volunteered (12 males, mean age $23 \pm 2.57$, range $18-28$ ) for this study. Inclusion criteria were: (1) age between 18 and 35 years; (2) no history of neurological disorders; (3) no history of substance abuse or dependence; (4) no current use of central nervous system-effective medication; (5) no history of brain surgery, tumor, or intracranial metal implantation; (6) no implant of cardiac pacemaker. This study was conducted in accordance to the ethical standards of the Declaration of Helsinki and was approved by a local ethics committee (SISNEP, Brazil; CAAE no. 390,272,000-08).

\subsection{Procedures}

The study was a double-blinded, randomized, sham-controlled and single-center study, where the participants were randomly assigned to one of three different stimulation conditions (leftanodal/right-cathodal, left-cathodal/right-anodal or sham). Prior to the experiment, all participants responded to a demographic data survey.

Initially, they were assessed with a visual analog mood scale (VAMS). Immediately after, they underwent tDCS stimulation during 5 min while resting. The stimulation continued during videos presentation (pain stimuli task) while the participant's pupil diameter was measured. After each video the participants evaluated it's emotional valence and arousal. When finishing this task, the tDCS was turned off and the volunteers responded once more to the VAMS and also a self-pain perception scale. The instruments of evaluation and procedures are described in detail.

\subsection{Emotional contagion for pain}

The task comprised 8 highly arousing pain video clips from the Emotional Movie Database [11]. The selected movies were from the Pain movies categories (no. 1000, 1001, 1002, 1004, 1005, 1006, $1007,1009)$, and the averaged validation rates for these stimuli were, in a 1 to 9 -point scale: valence $2 \pm 0.1$ and arousal $7 \pm 0.18$. The videos were presented using the software Nyan 2.0 (Interactive Minds $\mathrm{GmbH}$ ). Contrary to the majority of studies in pain empathy that used static images as stimuli, we used videos in order to make the pain scenes more real [12]. All videos consisted of painful situations and were $40 \mathrm{~s}$ long and had no audio. After the video, participants were shown two screens in which valence and arousal ratings were assessed. Participants were asked to rate the emotional impact of the experience of seeing the video clip on a 1 to 9-point Self-Assessment Manikin scale [13]. All the videos were presented during tDCS stimulation.

\subsection{Visual analog mood scale (VAMS)}

Before and after the study all participants were assessed using a visual analog mood scale (VAMS), which uses 5 different mood domains: alert/drowsy; confused/lucid; attentive/neglectful; happy/sad; hostile/friendly. Participants were asked to rate each domain from 0 to 9 (0 corresponding to $100 \%$ on one pole and 9 corresponding to $100 \%$ of the other pole [14,15].

\subsection{Self-pain perception assessment - pain-related words}

Immediately after watching all the clips and tDCS stimulation, participants were asked to respond to a subjective self-pain assessment. In this task, participants were requested to rate how much the following words described their feelings after the film clips: tortured, hurt and sore, using a 7-point scale (1 - nothing/7 - too much).

Finally, participants were asked to rate their discomfort with tDCS responding to a questionnaire, which screens for potential adverse effects.

\subsection{Pupil dilation assessment and analysis}

Pupil dilation data was registered $220 \mathrm{~ms}$ before video onset (i.e., baseline) and during film clips with EAS Binocular Series eyetracking system (interactive minds $\mathrm{GmbH}$ ) at $120 \mathrm{~Hz}$ (sampling rate) using the Nyan 2.0 software (interactive minds $\mathrm{GmbH}$ ). In order to control for possible environmental interference and pupillary response to light, the study was conducted in a dark and silent room, where participants were seated comfortably at a distance of $1 \mathrm{~m}$ from a 19-inch monitor (Lenovo ${ }^{\circledR}$ L197) with their head stabilized by an ophthalmologic chinrest. For pupil dilation analysis, the average pupil dilation for the whole video was obtained and corrected by its baseline. The baseline used was the pupil dilation response in the $100 \mathrm{~ms}$ prior to video exposition, during a fixation cross screen.

\subsection{Transcranial direct current stimulation}

Participants were randomly assigned to one of the three different stimulation conditions in a between-subjects design: anode on the left DLPFC (F3 according to the 10-20 EEG system) and cathode on the right DLPFC (F4); cathode on the left DLPFC and anode on 


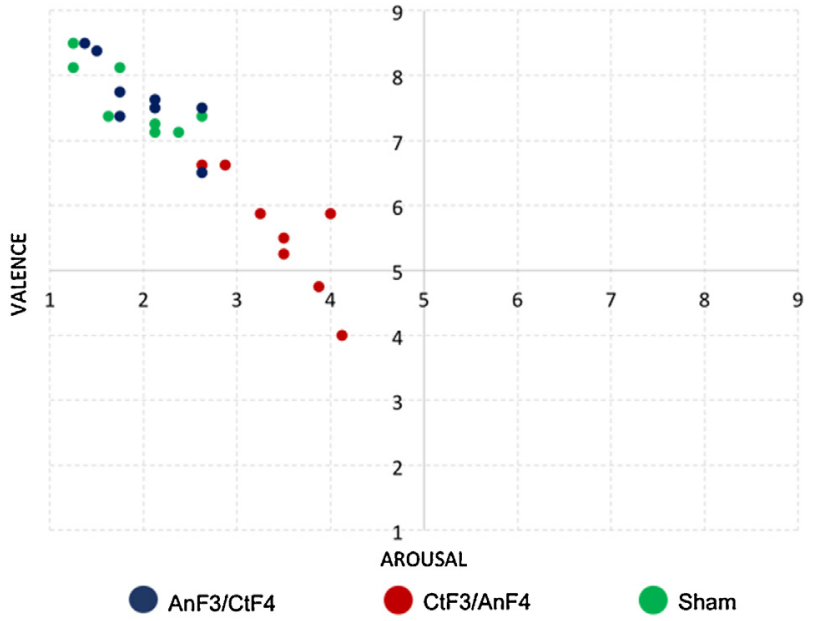

Fig. 1. TDCS effects on Valence and Arousal video evaluations.

Blue, red and green dots represent the mean rating of each video during anodal left/cathodal right, cathodal left/anodal right and sham stimulation, respectively. The vertical axis represents the arousal (being 1 very negative and 9 very positive). The horizontal axis represents the valence (being 1 not arousing and 9 very arousing). (For interpretation of the references to colour in this figure legend, the reader is referred to the web version of this article.)

the right DLPFC; or sham stimulation (same position as one of the active conditions, however the current was turned off after $30 \mathrm{~s}$ ). The tDCS positioning was defined in accordance to a previous study showing effective modulation in a pain empathy task [9]. All tDCS placement measures were in accordance with the 10-20 EEG positioning system [16]. A $2 \mathrm{~mA}$ direct current was delivered through a pair of $35 \mathrm{~cm}^{2}(5 \mathrm{~cm} \times 7 \mathrm{~cm})$ electrodes in saline soaked sponges; tDCS started 5 min prior to the experimental task, and throughout the entirety of the task (resulting in a total of approximately $15 \mathrm{~min}$ of stimulation).

\section{Results}

The entire procedure was well tolerated by all the participants and no adverse effects were reported. Participants only reported an initial and transient itching sensation.

Statistical analyses were carried out using SPSS software (version 20). Repeated-measures analysis of variance (ANOVA) was conducted to measure the impact of tDCS on scales evaluation and pupil dilatation. When appropriate, Fisher's Least Significant Difference (LSD) Post-Hoc tests were performed.

\subsection{Effect of tDCS on emotional valence and arousal assessment}

A mixed ANOVAs with valence scores as dependent variable, tDCS (left-anodal/right-cathodal, left-cathodal/right-anodal and sham) as between-subjects factor and Videos (8 movies) as within-subjects factor, revealed a significant main effect of Videos $\left(F_{7,147}=5.1, p<0.001, \eta p^{2}=0.2\right)$ and also of tDCS $\left(F_{2,21}=8.2, p=0.002, \eta p^{2}=0.4\right)$. No significant effect for the interaction between tDCS and Videos were found $\left(F_{14,147}=0.7, p=0.8\right.$, $\left.\eta p^{2}=0.06\right)$. Regarding the tDCS effect, LSD post-hoc indicated significant valence differences between left-cathodal/right-anodal in comparison with left-anodal/right-cathodal $(p=0.002)$ and sham $(p=0.003)$. As it can be seen in Fig. 1 and Table 1 , this effect was due to a decreased negative valence assessment during leftcathodal/right-anodal tDCS.

To analyze differences in emotional arousal, another mixed ANOVAs were conducted with arousal scores as the dependent variable, tDCS as between-subjects factor and Videos as within-subjects factors. A significant main effect of Videos $\left(F_{7,147}=4.7, p<0.001\right.$,
Table 1

Effect of tDCS on emotional valence and arousal assessment.

\begin{tabular}{|c|c|c|c|c|c|}
\hline \multirow{2}{*}{$\begin{array}{l}\text { Condition } \\
\text { AnF3/CtF4 }\end{array}$} & \multirow{2}{*}{$\begin{array}{l}\text { Movies } \\
1\end{array}$} & \multicolumn{2}{|c|}{$\begin{array}{l}\text { Valence } \\
\text { Mean }( \pm S E)\end{array}$} & \multicolumn{2}{|c|}{$\begin{array}{l}\text { Arousal } \\
\text { Mean }( \pm S E)\end{array}$} \\
\hline & & 1.75 & \pm 0.52 & 8.13 & \pm 0.71 \\
\hline & 2 & 1.25 & \pm 0.28 & 8.50 & \pm 0.53 \\
\hline & 3 & 2.38 & \pm 0.49 & 7.13 & \pm 0.63 \\
\hline & 4 & 1.63 & \pm 0.47 & 7.38 & \pm 0.65 \\
\hline & 5 & 2.63 & \pm 0.47 & 7.38 & \pm 0.50 \\
\hline & 6 & 1.25 & \pm 0.42 & 8.13 & \pm 0.52 \\
\hline & 7 & 2.13 & \pm 0.42 & 7.25 & \pm 0.52 \\
\hline & 8 & 2.13 & \pm 0.46 & 7.13 & \pm 0.80 \\
\hline \multirow[t]{8}{*}{$\mathrm{CtF} 3 / \mathrm{AnF} 4$} & 1 & 4.00 & \pm 0.52 & 5.88 & \pm 0.71 \\
\hline & 2 & 2.63 & \pm 0.28 & 6.63 & \pm 0.53 \\
\hline & 3 & 4.13 & \pm 0.49 & 4.00 & \pm 0.63 \\
\hline & 4 & 3.50 & \pm 0.47 & 5.50 & \pm 0.65 \\
\hline & 5 & 3.88 & \pm 0.47 & 4.75 & \pm 0.50 \\
\hline & 6 & 2.88 & \pm 0.42 & 6.63 & \pm 0.52 \\
\hline & 7 & 3.25 & \pm 0.42 & 5.88 & \pm 0.52 \\
\hline & 8 & 3.50 & \pm 0.46 & 5.25 & \pm 0.80 \\
\hline \multirow[t]{8}{*}{ Sham } & 1 & 1.75 & \pm 0.52 & 7.38 & \pm 0.71 \\
\hline & 2 & 1.38 & \pm 0.28 & 8.50 & \pm 0.53 \\
\hline & 3 & 2.63 & \pm 0.49 & 7.50 & \pm 0.63 \\
\hline & 4 & 1.50 & \pm 0.47 & 8.38 & \pm 0.65 \\
\hline & 5 & 2.63 & \pm 0.47 & 6.50 & \pm 0.50 \\
\hline & 6 & 2.13 & \pm 0.42 & 7.50 & \pm 0.52 \\
\hline & 7 & 1.75 & \pm 0.42 & 7.75 & \pm 0.52 \\
\hline & 8 & 2.13 & \pm 0.46 & 7.63 & \pm 0.80 \\
\hline
\end{tabular}

Values are described as mean $+/$ - SE.

$\left.\eta p^{2}=0.2\right)$ and $\operatorname{tDCS}\left(F_{2,21}=7.3, p=0.004, \eta p^{2}=0.4\right)$ was found. Similar to the above results, no significant effects were found for the interaction tDCS and Videos $\left(F_{14,147}=1.2, p=0.3, \eta p^{2}=0.1\right)$. With regard to the tDCS effect, LSD post-hoc revealed significant differences between left-cathodal/right-anodal in comparison with left-anodal/right-cathodal $(p=0.004)$ and $\operatorname{sham}(p=0.003)$. As it can be seen in Fig. 1, this effect was due to a decreased arousal response during left-cathodal/right-anodal stimulation.

During left-cathodal/right-anodal condition, participants presented decreased negative valence evaluation by $80 \pm 36 \%$ and $90 \pm 34 \%$ in comparison with sham and left-anodal/right-cathodal conditions, respectively, and decreased arousal evaluation by $27 \pm 11 \%$ and $27 \pm 9 \%$ in comparison with sham and leftanodal/right-cathodal conditions, respectively.

\subsection{Effects of $t D C S$ on pupil dilation response to the pain videos}

A mixed ANOVAs with pupil dilation average as the dependent variable was performed, with the tDCS as between-subject factor and Videos as within-subjects factor revealed significant main effects of Pupil Dilatation for $\operatorname{tDCS}\left(F_{2,19}=3.5, p=0.049\right.$, $\left.\eta p^{2}=0.3\right)$ and Videos $\left(F_{7,133}=56.8, p<0.001, \eta p^{2}=0.8\right)$. Once again, no significant interaction between tDCS and Videos was found $\left(F_{14,133}=0.7, p=0.8, \eta p^{2}=0.07\right)$. With regard to tDCS, LSD post-hoc showed significant differences on pupil dilatation variation during left-cathodal/right-anodal in comparison with left-anodal/rightcathodal condition $(p=0.02)$ and trend to significant effect in comparison with sham condition $(p=0.07)$. These effects were due to a larger variation of pupil dilatation as compared to baseline for left-cathodal/right-anodal $(0.12 \pm 0.02)$ with respect to the observed variation for sham $(0.07 \pm 0.02)$ and left-anodal/rightcathodal $(0.06 \pm 0.02)$ conditions. 


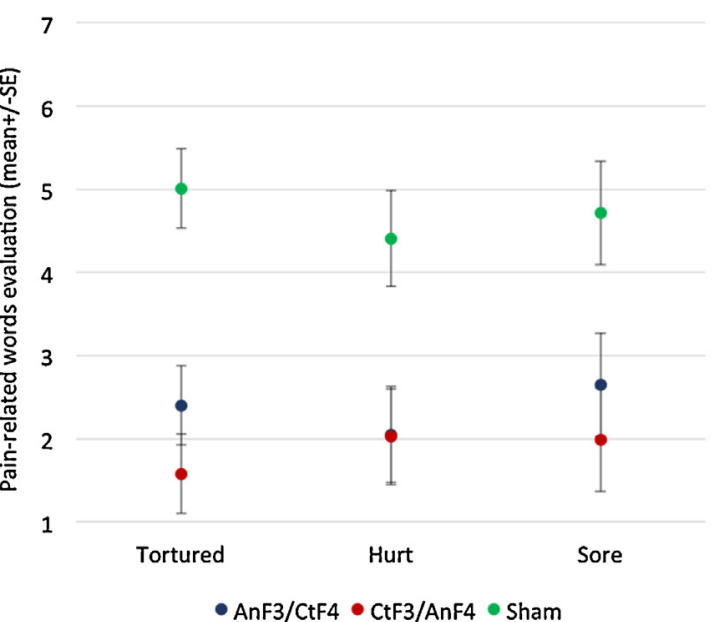

Fig. 2. TDCS effects over pain-related words.

Blue, red and green dots represent the mean rating of each word during anodal left/cathodal right, cathodal left/anodal right and sham stimulation, respectively. Bars represent SD. (For interpretation of the references to colour in this figure legend, the reader is referred to the web version of this article.)

\subsection{Effects of tDCS on subjective self-pain perception}

Mixed ANOVAs was conducted with self-pain perception score as the dependent variable, tDCS as between-subjects factor and Words (tortured, hurt and sore) as within-subjects factor. No significant effects for Words $\left(F_{2,42}=0.6, p=0.6, \eta p^{2}=0.03\right)$ and for the interaction between Words and tDCS was found $\left(F_{4,42}=0.8, p=0.5\right.$, $\left.\eta p^{2}=0.07\right)$. However, the ANOVAs revealed a significant main effect for tDCS $\left(F_{2,21}=9.5, p=0.001, \eta p^{2}=0.5\right)$. LSD post-hoc showed a significant difference between sham tDCS and left-anodal/rightcathodal $(p=0.003)$ and left-cathodal/right-anodal $(p=0.001)$. No significant difference was observed between the active conditions $(p=0.5)$. This effect was due to reduced scores of self-pain perception for both active conditions as compared to sham as illustrated in Fig. 2. .

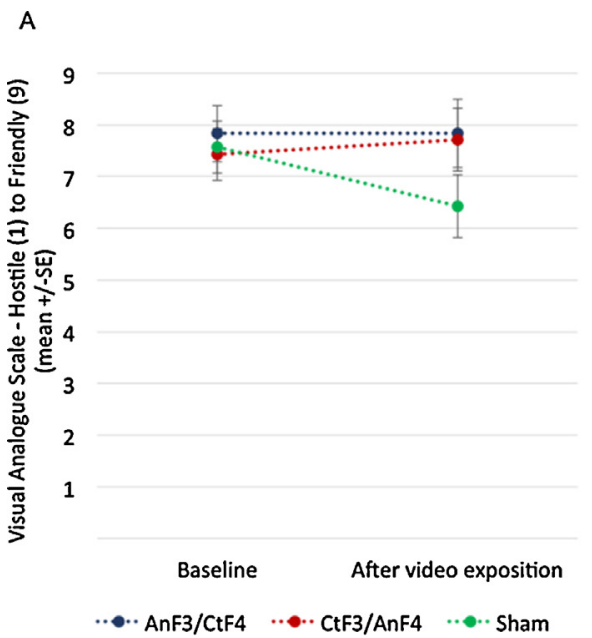

\subsection{Effect of tDCS on mood}

Mixed ANOVAs were conducted for each mood component considering the type of stimulation and Time (pre vs post tDCS). We found a significant interaction of tDCS and Time for hostile/friendly dimension $\left(F_{2,17}=4.13, p=0.04, \eta p^{2}=0.3\right)$ and a trend for tDCS and Time interaction effect for happy/sad dimension $\left(F_{2,17}=2.9\right.$, $p=0.08, \eta p^{2}=0.25$ ). As can be seen in Fig. 3, participants reported an increase on hostile feelings after sham condition as compared to the baseline $(p=0.007)$, while no differences were observed for both left-anodal/right-cathodal $(p=1.0)$ and left-cathodal/right-anodal $(p=0.5)$. No other significant effects were found for the remaining mood components.

\section{Discussion}

Our findings are consistent with our initial hypothesis that tDCSinduced modulation of DLPFC excitability changes the empathic affective responses for observed pain. Our findings can be divided into two main contributions. We first show that both active DLPFC stimulation conditions have a similar effect on self-pain perception and mood response (indexed by hostility). This effect is consistent with previous literature showing that both left and right DLPFC have similar roles in emotion regulation [5]. In the same line, the general response of decreased self-pain perception when seeing other's in pain agrees with neuromodulation studies showing that tDCS-induced imbalance of left and right DLPFC is associated with a decreased self-pain perception when receiving painful stimuli [9]. Specifically, Boggio et al. [3] found that left DLPFC activity enhancement results in increased pain threshold. Additionally, Boggio et al. [9] found that left DLPFC anodal stimulation decreased self-discomfort and unpleasantness judgments of pictures from people with injuries. These categories assess self-oriented reactions linked to affective/motivational component of pain when seeing others in painful situations [2]. More recently, another study by Brunoni et al. [17] showed that anodal left/cathodal right stimulation was associated with decreased heart rate variability and salivary cortisol levels in response to negative stimuli. In summary, our findings suggest that tDCS-induced DLPFC lateralized activity, regardless of the direction, is associated with a general affective modulation.

B

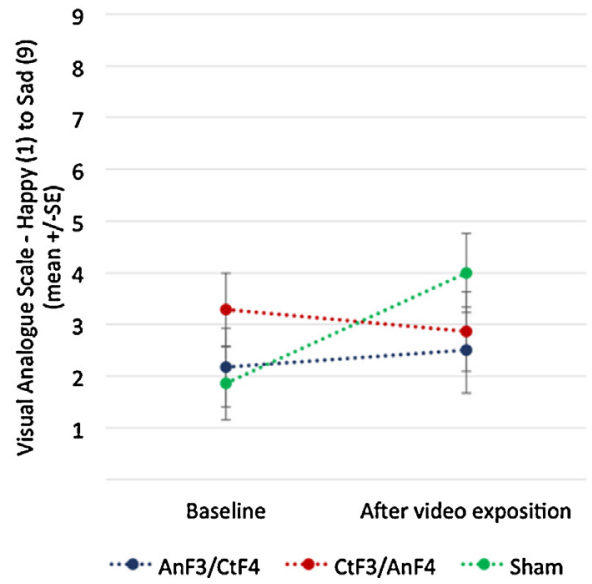

Fig. 3. TDCS effects over mood scales.

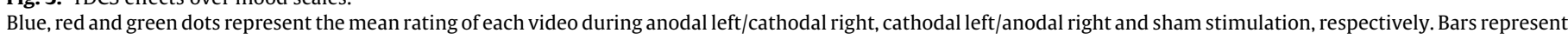

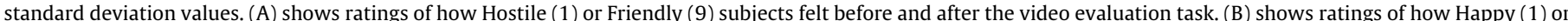

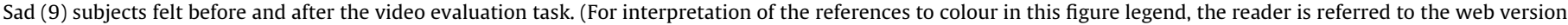
of this article.) 
The second important contribution of this study was to show that though both left and right DLPFC areas are involved in emotional regulation of pain, we observed a specific effect for cathodal left/anodal right stimulation on valence and arousal. This condition of stimulation was associated with decreased valence and arousal for the pain empathy stimuli. A possible explanation for this effect could be speculated from recent findings, which showed specific hemispheric roles for emotional regulation. According to Ochsner et al. [5], neuroimaging studies have shown that left hemisphere is involved in both increasing and decreasing emotional responses through cognitive reappraisal, whereas, the right hemisphere is involved with decreased emotional response only (but to a greater extent when compared to the left). The cognitive reappraisal tactics are also proposed to be different between hemispheres: the left DLPFC appears to be more active in reinterpreting or drifting attention from negative stimulus, whereas, the right is more related to psychological distancing techniques [5].

Therefore, we can hypothesize that the right DLPFC anodal stimulation may have led to a stronger down-regulation of empathic pain through the psychological distancing of the negative stimuli, leading to a decreased self-pain perception and decreased perception of intensity and valence of the videos. Meanwhile, the left hemisphere anodal stimulation may have acted on emotional regulation through a different mechanism that regulated how other's pain affects the self, but maintaining the cognitive perception of the videos as highly negative and intense. Pupillary response data seems to support this hypothesis, since the increased pupil dilation during cathodal left/anodal right may reflect higher cognitive demands due to reappraisal processes [10]. Nonetheless, future studies should address this hypothesis by evaluating the strategies used to cope with the stimuli.

Our study has some limitations. First, we used the 10-10 EEG system to localize the DLPFC. A recent study has proposed the use of an alternate method for DLPFC localization, the Omini-LateralElectrode-System (OLE System) [18]. This study shows that the OLE system reduces between subject current flow variability. It is unclear whether this system would also enhance behavioral results, but this should be further explored in future studies. In addition, it is also important to consider the possibility that our findings could have been due to indirect effects of tDCS over other areas (including pain matrix) besides the DLPFC. Future studies combining neuroimaging and neuromodulation methods can test this mechanistic hypothesis. Another limitation is the lack of neurophysiological data to support potential mechanisms explaining our data. The classical notion of a broad excitation under the anode electrode and inhibition under the cathode electrode has been challenged by recent studies [19]. For instance, it has been shown that different current intensities can result in an opposite effect on cortical excitability [19], and also on behavioral responses [6]. Therefore, further studies should also investigate dose-dependent response.

\section{Conclusion}

This study helps clarify the differential role of DLPFC hemispheres on different components of pain perception. Our findings further suggest a bilateral DLPFC involvement on self-regulation and a differential role of the right DLPFC on valence/arousal evaluation for other's pain. These findings, therefore, advance further our understanding of the network involved in the emotional processing of affective empathy for pain. Furthermore, the novel insight that right and left DLPFC may affect pain perception through different mechanisms may also help to develop better markers to assess cognitive pain processing such as using event related potentials and also interventions based of focal modulation of cortical areas such as with the use of novel brain stimulation techniques. In fact, there is a need to develop better interventions and find novel targets for neuromodulatory treatments for pain. One of the areas of investigation is the target of neural areas related to affective processing. Our study provides, therefore, initial data that could be helpful for development of novel targets for brain stimulation.

\section{Acknowledgments}

This experiment was supported by the National Council for Scientific and Technological Development (CNPq-480891/2012$5)$. LMM is supported by a scientific initiation grant (CNPq 160553/2013-0). OML is supported by a FAPESP PhD grant (FAPESP - 2012/24696-1). PSB is a CNPq research fellow (304164/2012-7).

\section{References}

[1] L. van der Heiden, S. Scherpiet, L. Konicar, N. Birbaumer, R. Veit, Inter-individual differences in successful perspective taking during pain perception mediates emotional responsiveness in self and others: an fMRI study, Neuroimage 65 (2015) 387-394.

[2] T. Singer, B. Seymour, J. O'Doherty, H. Kaube, R.J. Dolan, C.D. Frith, Empathy for pain involves the affective but not sensory components of pain, Science 303 (5661) (2004) 1157-1162.

[3] P.S. Boggio, S. Zaghi, M. Lopes, F. Fregni, Modulatory effects of anodal transcranial direct current stimulation on perception and pain thresholds in healthy volunteers, Eur. J. Neurol. 15 (October (10)) (2008) 1124-1130.

[4] J. Lorenz, S. Minoshima, K.L. Casey, Keeping pain out of mind: the role of the dorsolateral prefrontal cortex in pain modulation, Brain 126 (5) (2003) 1079-1091.

[5] K.N. Ochsner, J.A. Silvers, J.T. Buhle, Functional imaging studies of emotion regulation: a synthetic review and evolving model of the cognitive control of emotion, Ann. N.Y. Acad. Sci. 1251 (2012).

[6] P.S. Boggio, R. Ferrucci, S.P. Rigonatti, P. Covre, M.A. Nitsche, A. Pascual-Leone, F. Fregni, Effects of transcranial direct current stimulation on working memory in patients with Parkinson's disease, J. Neurol. Sci. 249 (November (1)) (2006) 31-38.

[7] P.S. Boggio, C. Campanhã, C.A. Valasek, S. Fecteau, A. Pascual-Leone, F. Fregni, Modulation of decision-making in a gambling task in older adults with transcranial direct current stimulation, Eur. J. Neurosci. 31 (Feburary (3)) (2010) 593-597.

[8] M.A. Nitsche, L.G. Cohen, E.M. Wassermann, A. Priori, N. Lang, A. Antal, W. Paulus, F. Hummel, P.S. Boggio, F. Fregni, A. Pascual-Leone, Transcranial direct current stimulation: state of the art 2008, Brain Stimul. 1 (July (3)) (2008) 206-223.

[9] P.S. Boggio, S. Zaghi, F. Fregni, Modulation of emotions associated with images of human pain using anodal transcranial direct current stimulation (tDCS), Neuropsychologia 47 (Janurary (1)) (2009) 212-217.

[10] E. Granholm, S.R. Steinhauer, Pupillometric measures of cognitive and emotional processes, Int. J Psychophysiol. 52 (March (1)) (2004) 1-6.

[11] S. Carvalho, J. Leite, S. Galdo-Álvarez, O.F. Gonçalves, The Emotional Movie Database (EMDB): a self-report and psychophysiological study, Appl. Psychophysiol. Biofeedback 37 (December (4)) (2012) 279-294.

[12] G. Recio, W. Sommer, A. Schacht, Electrophysiological correlates of perceiving and evaluating static and dynamic facial emotional expressions, Brain Res. 1376 (February) (2011) 66-75.

[13] M. Bradley, P.J. Lang, Measuring emotion: the self-Assesment manikin and the semantic diferential, J. Behav. Ther. Exp. Psychiatry 25 (1) (1994).

[14] F. Fregni, P. Liguori, S. Fecteau, M.A. Nitsche, A. Pascual-Leone, P.S. Boggio, Cortical stimulation of the prefrontal cortex with transcranial direct current stimulation reduces cue-provoked smoking craving: a randomized, sham-controlled study, J. Clin. Psychiatry 69 (1) (2008) 32-40.

[15] F. Fregni, F. Orsati, W. Pedrosa, S. Fecteau, F.A.M. Tome, M.A. Nitsche, P.S. Boggio, Transcranial direct current stimulation of the prefrontal cortex modulates the desire for specific foods, Appetite 51 (1) (2008) 34-41.

[16] H.H. Jasper, The ten twenty electrode system of the international federation, Electroencephalogr. Clin. Neurophysiol. 10 (1958) 371-375.

[17] A.R. Brunoni, A.H. Kemp, E.M. Dantas, A.C. Goulart, M.A. Nunes, P.S. Boggio, J.G. Mill, P.A. Lotufo, F. Fregni, I.M. Benseñor, Heart rate variability is a trait marker of major depressive disorder: evidence from the sertraline vs. electric current therapy to treat depression clinical study, Int. J. Neuropsychopharmacol. 16 (October (9)) (2013) 1937-1949.

[18] O. Seibt, A.R. Brunoni, Y. Huang, M. Bikson, The pursuit of DLPFC: non-neuronavigated methods to target the left dorsolateral pre-frontal cortex with symmetric bicephalic transcranial direct current stimulation (tDCS), Brain Stimulat. (2015), Available online, 16 Januarary 2015 http://dx.doi.org/10.1016/j.brs.2015.01.401

[19] G. Batsikadze, V. Moliadze, W. Paulus, M. Kuo, M.A. Nitsche, Partially non-linear stimulation intensity-dependent effects of direct current stimulation on motor cortex excitability in humans, J. Physiol. 59 (17) (2013) 1987-2000. 